\title{
A Robust Control Method for Lateral Stability Control of In-Wheel Motored Electric Vehicle Based on Sideslip Angle Observer
}

\author{
Yaxiong Wang, ${ }^{1}$ Feng Kang $\mathbb{D}$, 1 Taipeng Wang, ${ }^{2}$ and Hongbin Ren $\mathbb{D}^{2}$ \\ ${ }^{1}$ School of Technology, Beijing Forestry University, 35 Qinghua East Road, Beijing 100083, China \\ ${ }^{2}$ School of Mechanical Engineering, Beijing Institute of Technology, Beijing 100081, China \\ Correspondence should be addressed to Feng Kang; kangfeng98@bjfu.edu.cn
}

Received 27 August 2017; Revised 16 November 2017; Accepted 5 December 2017; Published 7 February 2018

Academic Editor: Mario Terzo

Copyright ( $) 2018$ Yaxiong Wang et al. This is an open access article distributed under the Creative Commons Attribution License, which permits unrestricted use, distribution, and reproduction in any medium, provided the original work is properly cited.

In-wheel motored powertrain on electric vehicles has more potential in maneuverability and active safety control. This paper investigates the longitudinal and lateral integrated control through the active front steering and yaw moment control systems considering the saturation characteristics of tire forces. To obtain the vehicle sideslip angle of mass center, the virtual lateral tire force sensors are designed based on the unscented Kalman filtering (UKF). And the sideslip angle is estimated by using the dynamicsbased approaches. Moreover, based on the estimated vehicle state information, an upper level control system by using robust control theory is proposed to specify a desired yaw moment and correction front steering angle to work on the electric vehicles. The robustness of proposed algorithm is also analyzed. The wheel torques are distributed optimally by the wheel torque distribution control algorithm. Numerical simulation is carried out in Matlab/Simulink-Carsim cosimulation environment to demonstrate the effectiveness of the designed robust control algorithm for lateral stability control of in-wheel motored vehicle.

\section{Introduction}

In recent years, active safety systems have been developed and commercialized aiming at improving vehicle high speed safety and making drivers more aware of the situation around them. The objects of these systems are to keep the vehicle stable under extreme driving conditions and follow the driver's intention. All body motion control and disturbance forces as well as aerodynamic forces applied to the vehicle are generated in the contact patches between the tire and road surfaces. The maximum tire friction forces are determined by the road surface conditions and tire vertical forces. The summations of longitudinal and lateral tire forces cannot exceed the boundary of the tire friction ellipse. In-wheel motor EVs have many advantages as a platform for vehicle motion control in the viewpoint of vehicle stability control [1-4]. Motors are mounted inside each wheel and driven independently, and this brings more potential in the improvement of vehicle handling and stability performance because of its fast response with precise control and high efficiency.
The traction and brake forces of each individual wheel can be independently controlled by means of controlling the corresponding electric drive motor [5]. Active safety systems can significantly prevent vehicle accident or relieve drivers' workload. And with the in-wheel motor powertrain system, the advanced driving assistance system (ABS, braking assistance, electronic stability program (ESP), etc.) could be realized by software without additional mechanical or hydraulic components with greater reliability and increased safety [6]. In-wheel motored cars are capable of offering more passenger space than traditional engine-powered cars [7]. It is necessary to optimize the tire forces distribution and make the forces exerted by all tires efficiently, so as to keep them inside the tire friction ellipse. Additionally, with stringent vehicle emission regulation and fast development of motor and battery technologies, the in-wheel motor powertrain has become one of the most popular configurations in the area of alternative energy vehicles (AEVs) and are currently being developed by various car manufacturers [8-10]. AEVs have better mobility and fuel economy performances compared 
to the conventional internal combustion engine vehicles [11-13].

Extensive research has been conducted by using different control methodologies for in-wheel motored vehicle control systems in recent years [14-16]. Zhao et al. [17] proposed the model predictive control allocation for yaw stability improvement of four-wheel drive electric vehicles in critical driving conditions. The proposed allocation strategy obtained a desired yaw moment to apply on the EVs by the upper level control system, while the control allocation is used to determine control inputs for four driving motors by commanding appropriate wheel slips. A correctional linear quadratic regulator combined the feedback and feed forward control algorithm is introduced by $\mathrm{Li}$ et al. to deduce the object of the stability yaw moment in order to guarantee the yaw rate and sideslip angle stability. Her et al. [18] presented an integrated chassis control of the differential braking, the front and rear traction torques, and the active roll moment for optimized tire force coordination to enhance the limit handling performance. Zhang and Wang [19] presented a robust gain-scheduling approach for vehicle lateral dynamics control through active front steering and direct yaw moment control. van der Sande et al. [20] proposed robust control analysis for a steer-by-wire vehicle considering the nonlinear characteristics of tire forces. Li studied the active front steering and direct yaw control coordination control problem based on model predictive control theory. Wu et al. [21] proposed an improved robust internal model control algorithm blending model tracking and internal model control for active steering system in order to reach high performance of yaw rate tracking with certain robustness.

In order to improve the robust performance of vehicle lateral stability especially for high speed condition, a robust controller is designed in this paper, which is insensitive to external and internal disturbances and parameters variations, such as tire stiffness variation when tire forces are in saturation. Combining the sideslip angle observer, the robust controller is designed to generate the yaw moment to improve the longitudinal performances and lateral stability of the inwheel motored vehicle.

The paper is organized as follows: vehicle dynamic model and control reference model are presented in Section 2, while in Section 3, the sideslip angle estimation is designed for the controller, and system controller is designed and analyzed based on robust control theory. Tire forces distribution and in-wheel motor control are illustrated in Sections 4 and 5 , respectively; the efficiency of the proposed control is demonstrated by simulation results in Section 6 . At the end of this paper, the conclusion and future work are given.

\section{Vehicle Dynamic Modeling and Reference Model}

The single-track model is widely used in the study of vehicle lateral dynamics. The single-track model is also called bicycle model, as shown in Figure 1, which considers lateral and yaw motions under the assumption of negligible lateral load transfer, roll, and compliance steer while traveling on smooth road with constant speed. One of the main effects

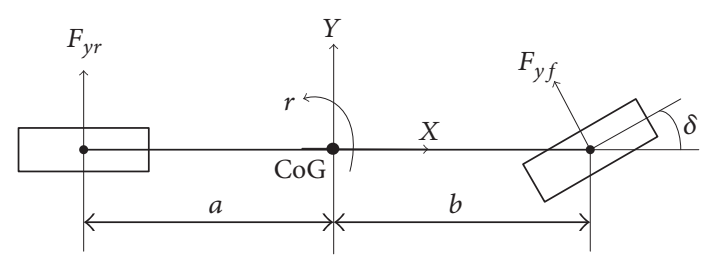

Figure 1: Dynamic bicycle model.

taken into account is the tire lateral slip phenomenon. However, the other main effects regarding vehicle behavior (such as axle deformation, axle kinematics, and suspension elastokinematics effect) are not modeled. The lateral tire force can be approximated as linear relationship with tire slip angle when vehicle lateral acceleration is less than $0.3 \mathrm{~g}$. So it is fairly accurate for the linear bicycle model, and the lateral tire force generation falls into the linear range. As tire slip angle increases due to a large steering maneuver which results in large lateral acceleration, the model will lose the fidelity compared to those responses of actual vehicle [22]. In this paper, a single-track vehicle model considering the tire forces saturation is used for state estimation and stability control.

The bicycle model can be expressed as

$$
\begin{aligned}
& \dot{v}=\frac{\left(F_{y f} \cos \delta_{f}+F_{y r}\right)}{m}-r u, \\
& \dot{r}=\frac{\left(a F_{y f} \cos \delta_{f}+b F_{y r}+\Delta T\right)}{I_{z}},
\end{aligned}
$$

where $v$ is lateral velocity; $r$ is yaw rate; $\delta_{f}$ is steering angle input; $\Delta T$ is yaw control torque.

$$
F_{y}=k_{i} \alpha_{i} \quad(i=f, r)
$$

With front tire stiffness, $k_{f} \in\left[k_{f \min }, k_{f \max }\right]$, and with rear tire stiffness, $k_{r} \in\left[k_{r \min }, k_{r \max }\right]$, where the subscripts $f$ and $r$ denote front axle and rear axle and $\alpha$ is tire lateral slip angle.

The sideslip angle of mass center is

$$
\begin{aligned}
\beta & =\frac{v}{u}, \\
a_{x} & =\dot{u}-r v, \\
a_{y} & =\dot{v}+r u,
\end{aligned}
$$

where $a_{x}, a_{y}$ are longitudinal and lateral acceleration of vehicle mass center, respectively.

For simplicity, a neutral steering model (i.e., $b k_{r}-a k_{f}=$ 0 ) is chosen as reference model. The state space equations of linear bicycle model can be expressed as

$$
\dot{X}_{\text {ref }}=A_{\text {ref }} X_{\text {ref }}+B_{\text {ref }} U \text {, }
$$




$$
\begin{aligned}
{\left[\begin{array}{c}
\dot{v} \\
\dot{r}
\end{array}\right]=} & {\left[\begin{array}{cc}
-\frac{k_{f}+k_{r}}{m u} & -1 \\
0 & -\frac{a^{2} k_{f}+b^{2} k_{r}}{I_{z} u}
\end{array}\right]\left[\begin{array}{l}
v \\
r
\end{array}\right] } \\
& +\left[\begin{array}{cc}
\frac{k_{r}}{m} & 0 \\
\frac{a k_{f}}{I_{z}} & \frac{1}{I_{z}}
\end{array}\right]\left[\begin{array}{c}
\delta \\
\Delta T
\end{array}\right] .
\end{aligned}
$$

The transfer functions can be deduced as

$$
\begin{aligned}
G_{\delta \rightarrow r}(s)_{\text {ref }} & =\frac{a k_{f} u}{I_{z} u s+a^{2} k_{f}+b^{2} k_{r}}, \\
G_{\Delta T \rightarrow r}(s)_{\text {ref }} & =\frac{\Delta T}{I_{z} u s+a^{2} k_{f}+b^{2} k_{r}} .
\end{aligned}
$$

So, the reference yaw rate can be defined as

$$
r_{\text {ref }}=G_{\delta \rightarrow r}(s)_{\text {ref }} \delta(s)+G_{\Delta T \rightarrow r}(s)_{\text {ref }} \Delta T(s) .
$$

Because the yaw rate is mainly affected by the steering angle input and the contribution of $\Delta T$ is relatively small, (6) can be further simplified as

$$
r_{\text {ref }}=K G_{\delta \rightarrow r}(s)_{\text {ref }} \delta(s),
$$

where $K$ is correction coefficient which considers the effects from $\Delta T$ and the maximum lateral tire forces can be generated.

\section{Robust Controller Design}

Optimal control algorithms are widely used in implementation of the industry control, but it is not always tolerant to changes in the environment disturbances or the control system. A control system is robust if it is insensitive to differences between the actual system and the nominal model of the system which is used in the controller design [23]. These differences are considered as model uncertainty or perturbations. Application of the robust control theory is important to build the reliable and precise systems [24, 25]. A robust control algorithm is designed to improve the lateral stability for in-wheel EV combined with online sideslip angle observer, as shown in Figure 2.

\subsection{Sideslip Angle Observer Based on Unscented Kalman Filter.} From a practical point of view, sideslip angle is considered as a significant signal in assessment of the transient response of vehicle stability. In this section, the estimation method of sideslip angle of vehicle mass center is presented based on the virtual tire forces sensor, as shown in Figure 3. The vehicle state information is estimated by using the UKF, which was proposed by Julier and Uhlman [26]. Detailed explanations of the UKF theory can be found in Ref [27]. The flowchart of the sideslip angle estimator is presented in Figure 4.

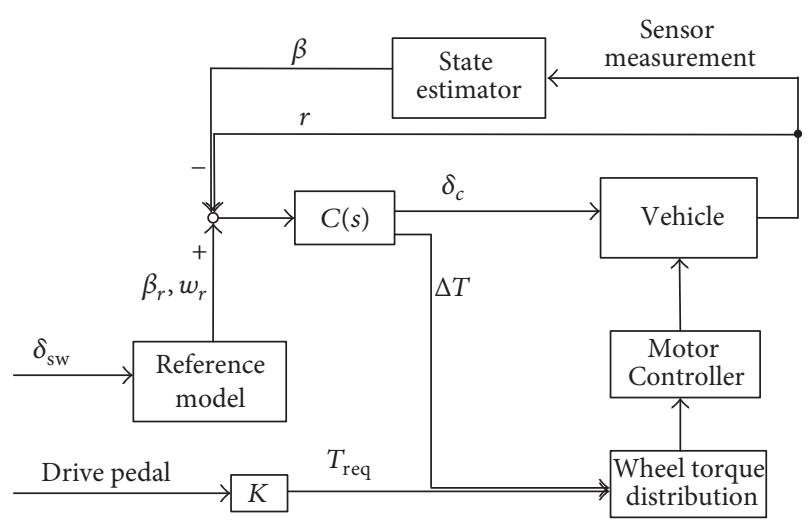

FIGURE 2: The diagram of the control logic.

Based on (1)-(3), the vehicle dynamic system can be rewritten in the discrete-time nonlinear state transition equation:

$$
\begin{gathered}
x(k+1)=f(x(k), u(k), w(k)), \\
y(k)=h(x(k), u(k), v(k)),
\end{gathered}
$$

where $f$ is the vehicle system dynamics; $x(k)$ is the state at the sampling instant $k, x=\left[u_{k}, v_{k}, r\right] ; u(k)$ is the input to the system at the sampling instant $k ; w(k)$ is the process noise which is assumed to be drawn from a zero mean with covariance $Q ; y(k)$ is a set of noisy measurements, $y=$ $\left[a_{x}, a_{y}\right] ; v(k)$ is the observation noise which is assumed to be zero mean Gaussian white noise with covariance $R$. The process of UKF can be summarized as follows:

(a) Sigma points' calculation: in this section, the sigma points are set by symmetrical sampling method. The mean of the vehicle state vector $x$ is $\bar{x}$ and the covariance is $P^{x}$. And the elements of sigma matrix $X=\left[x_{1}, x_{2}, \ldots, x_{k}, \ldots, x_{2 n+1}\right]$ can be generate by

$$
x_{k}= \begin{cases}\bar{x} & k=0, \\ \bar{x}+\left(\sqrt{(n+\lambda) P^{x}}\right)_{k} & k=1,2, \ldots, n, \\ \bar{x}-\left(\sqrt{(n+\lambda) P^{x}}\right)_{k-n} & k=n+1, \ldots, 2 n,\end{cases}
$$

where $\lambda=\alpha^{2}(n+\kappa)-n$ is the scaling parameter. The constant $\alpha$ determines the spread of the sigma points around $\bar{x}$ and is usually set as a small value $\left(10^{-4} \leq \alpha \leq 1\right) ; \kappa \geq 0$; it make sure that the covariance matrix is positively definite.

(b) These sigma vectors are updated by the system function

$$
X_{k+1}=X_{k+1}+f\left(X_{k+1}, u_{k}, w_{k}\right) \cdot \Delta T \text {. }
$$

(c) Measurement vector update is as follows:

$$
Y_{k+1}=h\left(x_{k+1}, u_{k+1}, v_{k+1}\right) \text {. }
$$




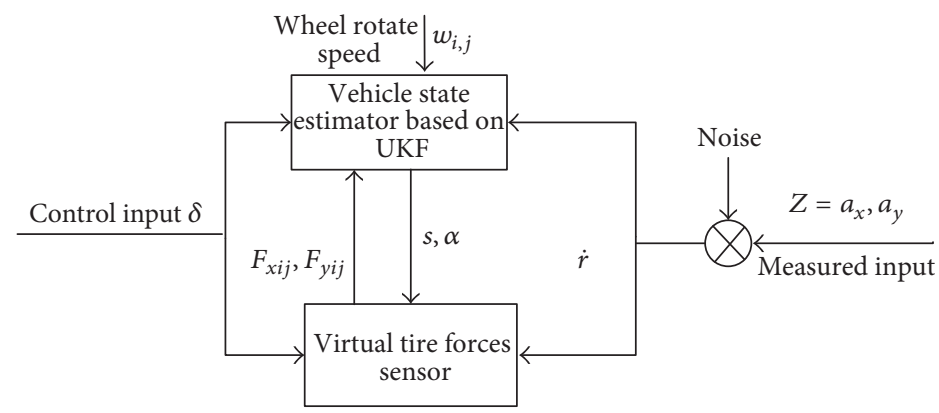

Figure 3: The flowchart of the sideslip angle estimator.

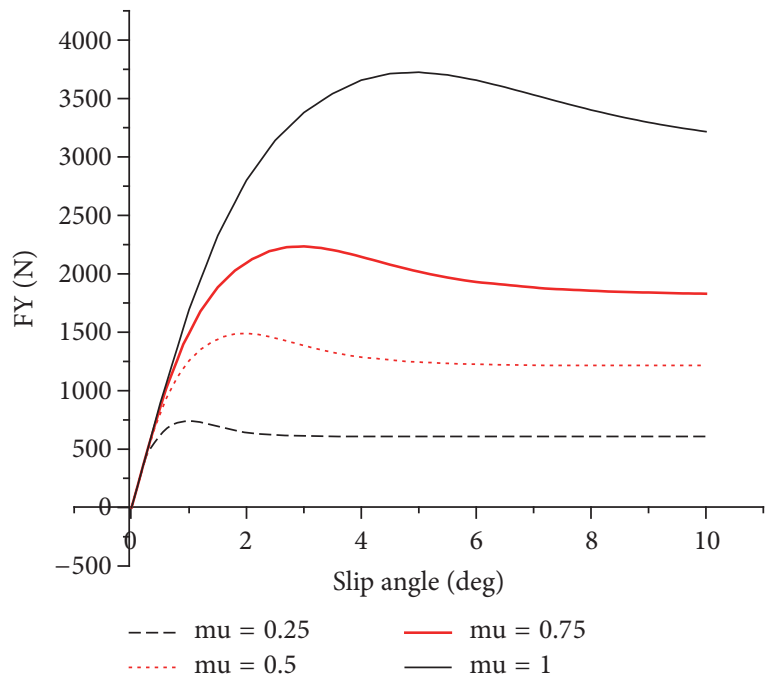

FIGURE 4: Lateral tire forces under different road friction coefficient.

The updated state vector $x_{k+1 \mid k}$ and the measurement value can be approximated by using a weighted sample of the sigma matrix,

$$
\begin{aligned}
x_{k+1 \mid k} & \approx \sum_{i=0}^{2 n}\left(W_{i}^{m}\left(X_{k+1}\right)_{i}\right), \\
y_{k+1} & \approx \sum_{i=0}^{2 n}\left(W_{i}^{m}\left(Y_{k+1}\right)_{i}\right) .
\end{aligned}
$$

(d) Covariance update: the covariance of the state vector and the measurement value can be calculated by

$$
\begin{aligned}
P_{k+1 \mid k}^{x}= & \sum_{i=0}^{2 n}\left[W_{i}^{c}\left(\left(X_{k+1}\right)_{i}-x_{k+1}\right)\left(\left(X_{k+1}\right)_{i}-x_{k+1}\right)^{\prime}\right] \\
& +Q
\end{aligned}
$$

$$
\begin{aligned}
P_{k+1}^{y}= & \sum_{i=0}^{2 n}\left[W_{i}^{c}\left(\left(Y_{k+1}\right)_{i}-y_{k+1}\right)\left(\left(Y_{k+1}\right)_{i}-y_{k+1}\right)^{\prime}\right] \\
& +R, \\
P_{k+1}^{x y}= & \sum_{i=0}^{2 n}\left[W_{i}^{c}\left(\left(X_{k+1}\right)_{i}-x_{k+1}\right)\left(\left(Y_{k+1}\right)_{i}-y_{k+1}\right)^{\prime}\right] .
\end{aligned}
$$

The weight can be acquired by

$$
\begin{aligned}
& W_{i}^{m}=[\frac{\lambda}{(n+\lambda)}, \underbrace{\frac{1 / 2}{(n+\lambda)}, \ldots, \frac{1 / 2}{(n+\lambda)}}_{2 n}], \\
& W_{i}^{c}=[\frac{\lambda}{(n+\lambda)}+1-\alpha^{2}+\gamma, \underbrace{\frac{1 / 2}{(n+\lambda)}, \ldots, \frac{1 / 2}{(n+\lambda)}}_{2 n}] \text {. }
\end{aligned}
$$

Assume that the system noise and the measurement noise are white Gaussian noise and covariance is $Q$ and $R$, respectively. $\gamma$ considers the high order moment of the prior distribution; for Gaussian distribution, here, $\gamma=2$ is optimal [27].

(e) The correction gain, system state vector, and covariance can be updated by

$$
\begin{aligned}
K_{k+1} & =P_{k+1}^{x y}\left(P_{k+1}^{y}\right)^{-1}, \\
x_{k+1 \mid k+1} & =x_{k+1 \mid k}+K_{k+1}\left(z_{k+1}-y_{k+1}\right), \\
P_{k+1 \mid k+1}^{x} & =P_{k+1 \mid k}^{x}-K_{k+1} P_{k+1}^{y}\left(K_{k+1}\right)^{\prime},
\end{aligned}
$$

where $z_{k+1}$ is the measurement value from the vehicle sensor.

3.2. Robust Controller Design. The linear bicycle model can be rewritten as

$$
\dot{X}=A X+B U
$$




$$
\begin{aligned}
& {\left[\begin{array}{c}
\dot{v} \\
\dot{r}
\end{array}\right]=\left[\begin{array}{cc}
-\frac{k_{f}+k_{r}}{m u} & \frac{-a k_{f}+b k_{r}}{m u}-1 \\
\frac{-a k_{f}+b k_{r}}{I_{z} u} & -\frac{a^{2} k_{f}+b^{2} k_{r}}{I_{z} u}
\end{array}\right]\left[\begin{array}{l}
v \\
r
\end{array}\right]+\left[\begin{array}{cc}
\frac{k_{r}}{m} & 0 \\
\frac{a k_{f}}{I_{z}} & \frac{1}{I_{z}}
\end{array}\right]\left[\begin{array}{c}
\delta_{f} \\
\Delta T
\end{array}\right],} \\
& y=r=C x, \\
& G_{p}(s)_{\delta \rightarrow r}=\frac{k_{f} u\left((a+b) k_{r}+a m u s\right)}{(a+b)^{2} k_{f} k_{r}+m\left(b k_{r}-a k_{f}\right) u^{2}+\left(\left(k_{f}+k_{r}\right) I_{z}+m\left(a^{2} k_{f}+b^{2} k_{r}\right)\right) u s+m I_{z} u^{2} s^{2}}, \\
& G_{p}(s)_{\Delta T \rightarrow r}=\frac{u\left(m u s+\left(k_{f}+k_{r}\right)\right)}{(a+b)^{2} k_{f} k_{r}+m\left(b k_{r}-a k_{f}\right) u^{2}+\left(\left(k_{f}+k_{r}\right) I_{z}+m\left(a^{2} k_{f}+b^{2} k_{r}\right)\right) u s+m I_{z} u^{2} s^{2}} .
\end{aligned}
$$

The zero poles can be deduced as

$$
\begin{aligned}
s_{0 \delta \rightarrow r} & =-\frac{(a+b) k_{r}}{a m u} ; \\
s_{0 \Delta T \rightarrow r} & =-\frac{k_{f}+k_{r}}{m u} .
\end{aligned}
$$

And the system frequency and damping ratio are

$$
\begin{aligned}
& f_{0}=\frac{1}{2 \pi} \sqrt{\frac{(a+b)^{2} k_{f} k_{r}+m\left(b k_{r}-a k_{f}\right) u^{2}}{m I_{z} u^{2}}}, \\
& \beta_{0}=\frac{\left(k_{f}+k_{r}\right) I_{z}+m\left(a^{2} k_{f}+b^{2} k_{r}\right)}{2 \sqrt{m I_{z}\left((a+b)^{2} k_{f} k_{r}+m\left(b k_{r}-a k_{f}\right) u^{2}\right)}} .
\end{aligned}
$$

For the unit step response, the steady state gain of yaw rate response is as follows:

$$
\begin{aligned}
& \underset{\substack{p \\
\operatorname{lims\rightarrow 0}_{\delta \rightarrow r}}}{G(s)^{2}}=\frac{(a+b) u k_{r} k_{f}}{(a+b)^{2} k_{f} k_{r}+\left(b k_{r}-a k_{f}\right) m u^{2}}, \\
& G_{p}(s)_{\Delta T \rightarrow r}=\frac{u\left(k_{f}+k_{r}\right)}{(a+b)^{2} k_{f} k_{r}+m\left(b k_{r}-a k_{f}\right) u^{2}} .
\end{aligned}
$$

The controller can be designed as

$$
\begin{gathered}
C_{1}(s)=k_{\delta \rightarrow r} \frac{1}{G_{p}(s)_{\delta \rightarrow r}} \frac{1}{1-s / s_{0 \delta \rightarrow r}} \\
\cdot \frac{s^{2} /\left(2 \pi f_{0}\right)^{2}+\left(\beta_{0} / \pi f_{0}\right) s+1}{1} \\
\cdot \frac{1}{s^{2} /\left(2 \pi f_{c}\right)^{2}+\left(\beta_{c} / \pi f_{c}\right) s+1}
\end{gathered}
$$

$$
\begin{gathered}
C_{2}(s)=k_{\Delta T \rightarrow r} \frac{1}{G_{p}(s)_{\Delta T \rightarrow r}} \frac{1}{1-s / s_{0 \Delta T \rightarrow r}} \\
\cdot \frac{s^{2} /\left(2 \pi f_{0}\right)^{2}+\left(\beta_{0} / \pi f_{0}\right) s+1}{1} \\
\cdot \frac{1}{s^{2} /\left(2 \pi f_{c}\right)^{2}+\left(\beta_{c} / \pi f_{c}\right) s+1},
\end{gathered}
$$

where $f_{c}$ and $\beta_{c}$ can be chosen freely for system pole assignment in order to improve the stability of the system. To improve the yaw rate response, we should increase the bandwidth of the controller, which means frequency $f_{c}>$ $f_{0}$. To suppress the vibration of second-order system, the damping coefficient should be larger than the controlled system, as $\beta_{c}>\beta_{0}$.

The open loop transfer function is

$$
\begin{aligned}
L_{n}(s) & =\left[\begin{array}{ll}
C_{1}(s) & \\
& C_{2}(s)
\end{array}\right]\left[\begin{array}{c}
G_{p}(s)_{\delta \rightarrow r} \\
G_{p}(s)_{\Delta T \rightarrow r}
\end{array}\right] \\
& =\left[\begin{array}{c}
C_{1}(s) G_{p}(s)_{\delta \rightarrow r} \\
C_{2}(s) G_{p}(s)_{\Delta T \rightarrow r}
\end{array}\right] \\
& =\left[\frac{k_{\delta \rightarrow r} /\left(2 \pi f_{c}\right)^{2}+\beta_{c} / \pi f_{c}+1}{k_{\Delta T \rightarrow r}}\right]=\left[\begin{array}{c}
L_{\delta \rightarrow r} \\
L_{\Delta T \rightarrow r}
\end{array}\right] .
\end{aligned}
$$

The close loop transfer function can be deduced as

$$
\begin{aligned}
& T_{n}(s)=\left[\begin{array}{ll}
\frac{L_{\delta \rightarrow r}}{L_{\delta \rightarrow r}+1} & \frac{L_{\Delta T \rightarrow r}}{L_{\Delta T \rightarrow r}+1}
\end{array}\right]^{\mathrm{T}},
\end{aligned}
$$

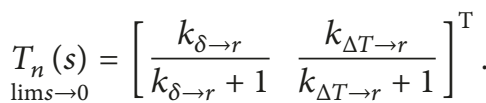

When the coefficient $\left[k_{\delta \rightarrow r} k_{\Delta T \rightarrow r}\right]$ is chosen sufficiently large, the steady state of close loop transfer function will go to 1 ; that is, the yaw rate can follow the reference yaw rate precisely. 


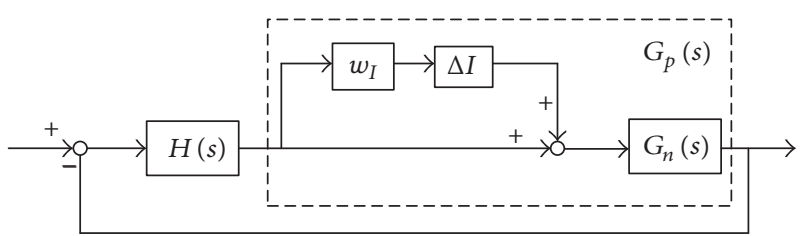

FIGURE 5: Feedback system with multiplicative uncertainty.

3.3. Robust Analysis. Vehicle stability heavily depends on the front and rear tire forces. It is appropriate to consider the effect of parameter-based uncertainty by using the $H_{\infty}$ analysis and design the controller to ensure that the system remains stable for all perturbations in the uncertain conditions.

The lateral tire force is increasing with the increase of tire slip angle, and when the slip angle is larger than a certain value, the tire force will be saturate, as shown in Figure 4. And the tire lateral stiffness varies with the increase of tire slip angle and tire road friction conditions. So we assume that there is multiplicative uncertain of magnitude $\left|w_{I}(s)\right|$. With the uncertainty, the loop transfer function becomes

$$
G_{p}(s)=G_{n}(s)\left(1+w_{I}(s) \Delta_{I}\right) \quad\left\|\Delta_{I}\right\|_{\infty} \leq 1,
$$

where the subscript $I$ denotes input; $G_{n}(s)$ is nominal system plant and always assumes that the close loop system is stable; $\Delta_{I}$ is system perturbations; $w_{I}$ is system weighting function. It is always difficult to obtain the system perturbations, and $\Delta_{I}$ will extend the perturbation range. In special case, $\left\|\Delta_{I}\right\|_{\infty}=1$ is the system perturbation is the worst case; we choose the weighting function $w_{I}(s)$ to avoid the perturbations from deviating the reality of system. Here $w_{I}(s)$ is any stable transfer function.

The open loop transfer function becomes

$$
\begin{aligned}
L_{p}(s) & =H(s) G_{p}(s)=G_{n}(s) H(s)\left(1+w_{I}(s) \Delta_{I}\right) \\
& =L_{n}+w_{I} L_{n} \Delta_{I},
\end{aligned}
$$

where $\Delta_{I}$ is the worst case of the system perturbation $\Delta_{I} \leq 1$ and $w_{I}$ is a weighting function of perturbation, as shown in Figure 5.

Considering the Nyquist plot of $L_{p}$, at each frequency find the smallest radius $l_{p}(w)$ which includes all the possible perturbation plant:

$$
\begin{aligned}
l_{p}(s) & =\max \left|\frac{G_{p}(j w)-G_{n}(j w)}{G_{n}(j w)}\right|=\max \left|w_{I}(s) \Delta_{I}\right| \\
& \leq\left|w_{I}(s)\right|, \quad \forall w .
\end{aligned}
$$

According to the Nyquist criterion [28], a feedback system is stable if and only if the contour in the $L_{p}(s)$-plant does not encircle the $(-1,0)$ point. The distance from nominal plant $L_{n}$ to point $(-1,0)$ must be bigger than the maximum perturbations $w_{I} L_{n}$; that is,

$$
\begin{aligned}
\left|w_{I} L_{n}\right| & <\left|L_{n}+1\right|, \quad \forall w, \\
\left|\frac{w_{I} L_{n}}{L_{n}+1}\right| & <1 \Longleftrightarrow\left\|w_{I} T_{n}\right\|_{\infty}<1, \quad \forall w .
\end{aligned}
$$

Combined with (27), the control system robust stability conditions can be deduced as

$$
\left[\begin{array}{ll}
\frac{k_{\delta \rightarrow r}}{k_{\delta \rightarrow r}+1} & \frac{k_{\Delta T \rightarrow r}}{k_{\Delta T \rightarrow r}+1}
\end{array}\right]^{\mathrm{T}}<\frac{1}{w_{I}}<\frac{1}{l_{p}(s)}
$$

where $l_{p}(s)=\left[l_{p 0 \delta \rightarrow r} l_{p 0 \Delta T \rightarrow r}\right]$.

$$
\begin{aligned}
& l_{p 0 \delta \rightarrow r}=l_{p}(s)_{\delta \rightarrow r}=m u^{2} \\
& \cdot \frac{a k_{f} k_{f \max }\left(k_{r}-k_{r \min }\right)+b k_{r} k_{r \min }\left(k_{f \max }-k_{f}\right)}{k_{r} k_{f}\left[(a+b)^{2} k_{f \max } k_{r \min }+\left(b k_{r \min }-a k_{f \max }\right) m u^{2}\right]}, \\
& l_{p 0 \Delta T \rightarrow r}=l_{p}(s)_{\Delta T \rightarrow r} \\
& =\frac{(a+b)^{2} k_{f} k_{r}+m\left(b k_{r}-a k_{f}\right) u^{2}}{(a+b)^{2} k_{f \max } k_{r \min }+m\left(b k_{r \min }-a k_{f \max }\right) u^{2}} \\
& \cdot \frac{k_{f \max }+k_{r \min }}{k_{f}+k_{r}}-1, \\
& l_{p}(s)_{\delta \rightarrow r}=\frac{k_{f \max }}{k_{f}}-1, \\
& l_{p}(s)_{\Delta T \rightarrow r}=0 . \\
& \operatorname{lims\rightarrow 0}
\end{aligned}
$$

\section{Tire Force Distribution}

The yaw stability mainly depends on the steering angle input and yaw moment control. And the active front steering control is directly input as yaw moment generator. The tire longitudinal tire forces can be calculated by

$$
\begin{aligned}
\Delta T_{c}= & \left(-B_{f} \cos \frac{\delta}{2}+a \sin \delta\right) F_{x f l}-\frac{B_{r} F_{x r l}}{2} \\
& +\left(B_{f} \cos \frac{\delta}{2}+a \sin \delta\right) F_{x f r}+\frac{B_{r} F_{x r r}}{2}, \\
\Delta T_{r}= & \sum T_{x i}, \\
\Delta T_{x i}= & F_{x i} \cdot R_{i},
\end{aligned}
$$

where $\Delta T_{c}$ is the corrected yaw moment generated from differential driving from motors; $\Delta T_{r}$ is total required driving torque from driver; $F_{x i}$ is the $i$ th longitudinal tire force; $R_{i}$ is the $i$ th tire roll radius.

When the slip angle is larger than the upper boundary, the tire forces will be in the saturation region, according to the friction circle theory [29]; the tire longitudinal and lateral forces follow the nonlinear constraint,

$$
F_{x i_{-} \text {sat }}^{2}+F_{y i_{-} \text {sat }}^{2} \leq(\mu F z)^{2} \text {, }
$$

where $\mu$ is the road friction coefficient. 


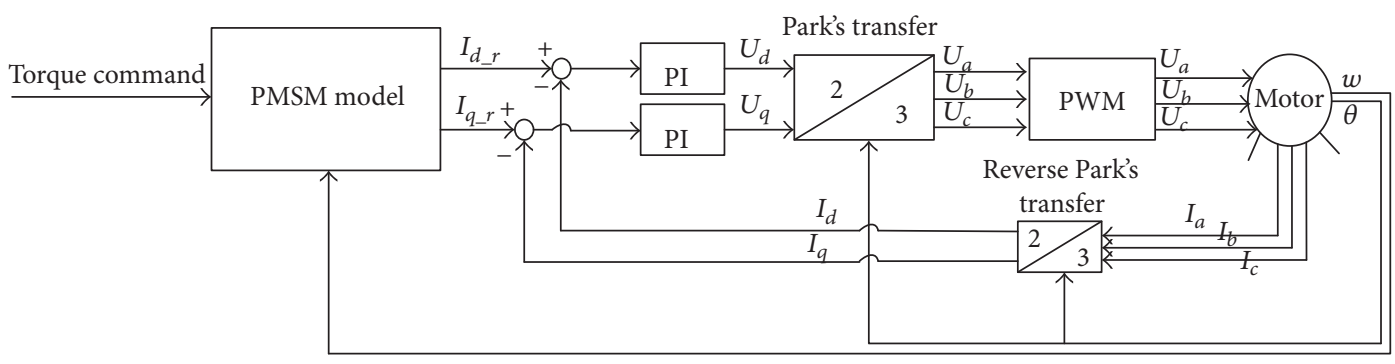

FIGURE 6: Control logic of permanent magnet synchronous motor.

So,

$$
0 \leq F_{x i \text { sat }} \leq \sqrt{(\mu F z)^{2}-F_{y i \text { sat }}^{2}} .
$$

Assuming that the torque split is 40 to 60 percent between front and rear axle, the four wheels driving torque can be obtained according to (34) (36) with consideration of the tire force friction circle constrain.

In order to improve the yaw stability of vehicle, the active front steering system and motor differential driving system work coordinately. The rule of the control coordination can be described as follows: when vehicle lateral acceleration is relative large, it means that tire tends to work close to the saturation zone. The values of yaw control torque will increase by differential longitudinal tire forces, so as to make a good use of tire forces. Likewise, in order to improve the longitudinal stability and traction ability of vehicle, when lateral acceleration is small, the active front steering will work.

\section{In-Wheel Motor Model and Control}

The function of the permanent-magnetic motor is as follows:

$$
\begin{aligned}
T_{e} & =\frac{1}{\tau s+1} T_{m}, \\
T_{m} & =K_{\text {motor }} i,
\end{aligned}
$$

where $\tau$ is the closed-loop response time which is a control characteristic of motor torque controller; $T_{m}$ is the motor driving torque command from control algorithm; $K_{\text {motor }}$ is the motor torque coefficient.

$$
V=R i+L i+K_{\text {cemf }} w
$$

where $V$ is the armature voltage, $R$ is the armature resistance, $i$ is the armature current, $w$ is the motor rotational speed, and $L$ is the armature inductance, $K_{\text {cemf }}$ is the counterelectromotive force coefficient. And the control logic of the permanent magnet synchronous motor (PMSM) is shown in Figure 6.

\section{Simulation and Discussion}

The sine-with-dwell maneuver is widely used to evaluate the performance of electronic stability control system [30].
TABLE 1: Vehicle parameters.

\begin{tabular}{lcc}
\hline Vehicle mass & $m$ & $1351 \mathrm{~kg}$ \\
Yaw moment of inertia & $I_{z}$ & $2031.41 \mathrm{kgm}^{2}$ \\
C.g. distance to front wheels & $a$ & $1.04 \mathrm{~m}$ \\
C.g. distance to rear wheels & $b$ & $1.56 \mathrm{~m}$ \\
Sprung c.g. height & $h_{\mathrm{cg}}$ & $0.542 \mathrm{~m}$ \\
Front track width & $B_{f}$ & $1481 \mathrm{~m}$ \\
Rear track width & $B_{r}$ & $1.486 \mathrm{~m}$ \\
The wheel radius & $R_{w}$ & $0.305 \mathrm{~m}$ \\
\hline
\end{tabular}

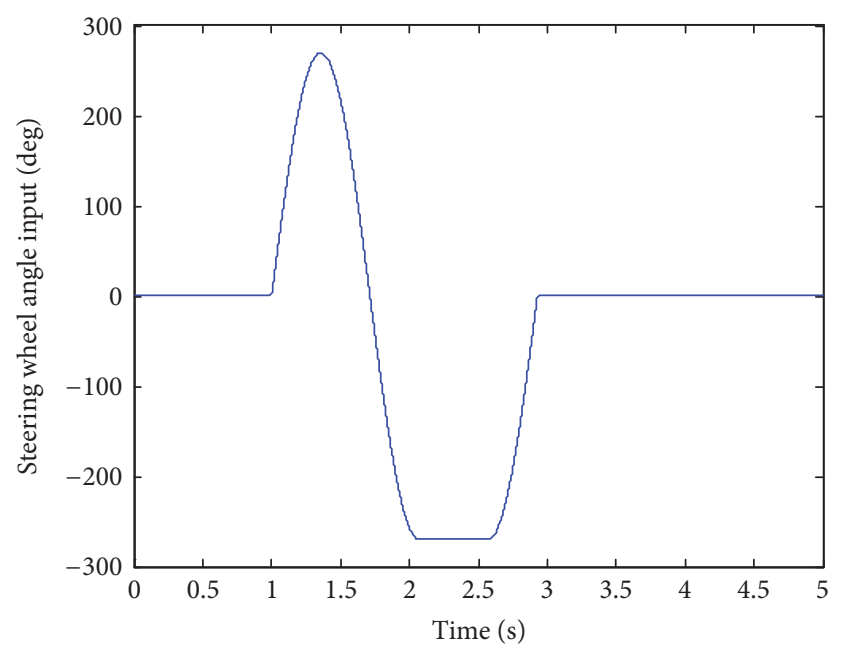

FIgURE 7: Sine-with-dwell steering wheel input.

In this section, in order to evaluate the performance of the stability control algorithm, the numerical simulation was implemented in the Matlab/Simulink environment and cosimulated with the Carsim software. Carsim is commercial vehicle dynamics software which is widely used in automotive industry. The performance of robust control is validated under sine-with-dwell maneuver. Figure 7 shows the steering wheel input angle of the sine-with-dwell maneuver. From this simulation scenario, all the dynamic characteristics of the vehicle can be adequately described, and the yaw stability of motor in-wheel EV can be fully verified. In the simulation, a vehicle runs at a constant speed of $50 \mathrm{~km} / \mathrm{h}$. The vehicle parameters are listed in Table 1. And the simulation results are plotted in Figures 7- 16. 

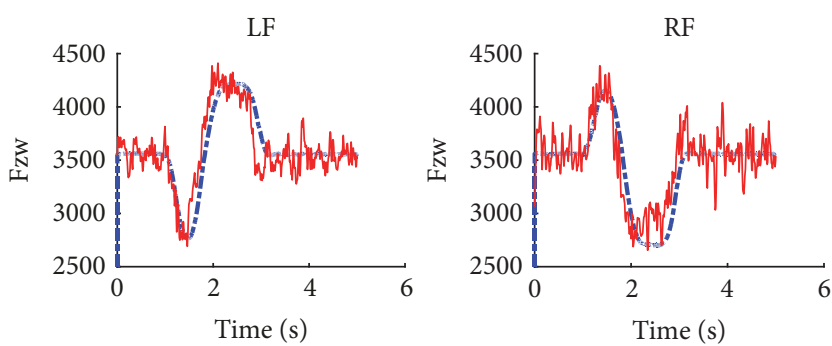

LR
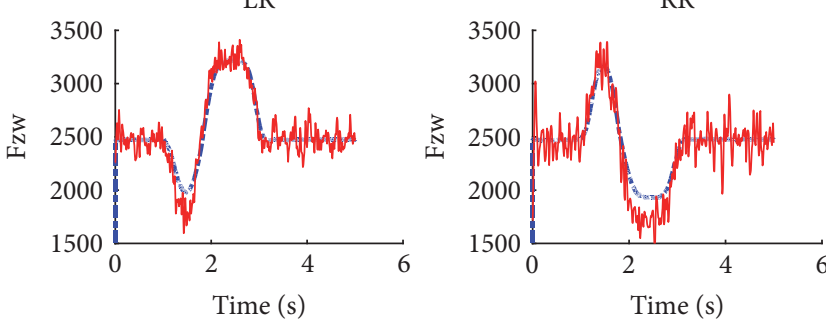

---- UKF

— Carsim

FIGURE 8: Estimation results comparison of vertical forces.
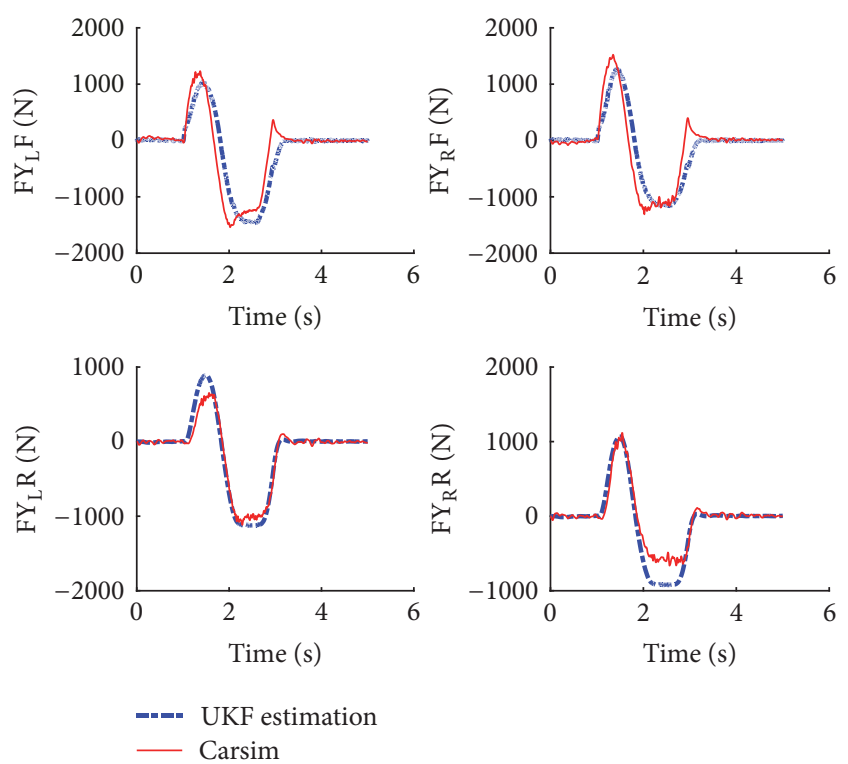

FIGURE 9: Estimation results comparison of tire lateral forces.

Under the sine-with-dwell simulation scenario, the robust control for vehicle stability combined with the wheel torque distribution strategy should not only meet the demand of vehicle steering maneuverability improvement but also improve the vehicle body stability under high speed condition. Yaw rate response is the maneuverability index, while the sideslip angle of mass center is an important index for vehicle stability [31].

In Figure 11, it can be clearly observed that compared with no control, the vehicle with the robust control strategy could follow the driver's intention perfectly, which means the maneuverability of controlled vehicle is improved significantly. Figure 12 compared the sideslip angle of mass center; we can find that the designed estimation strategy

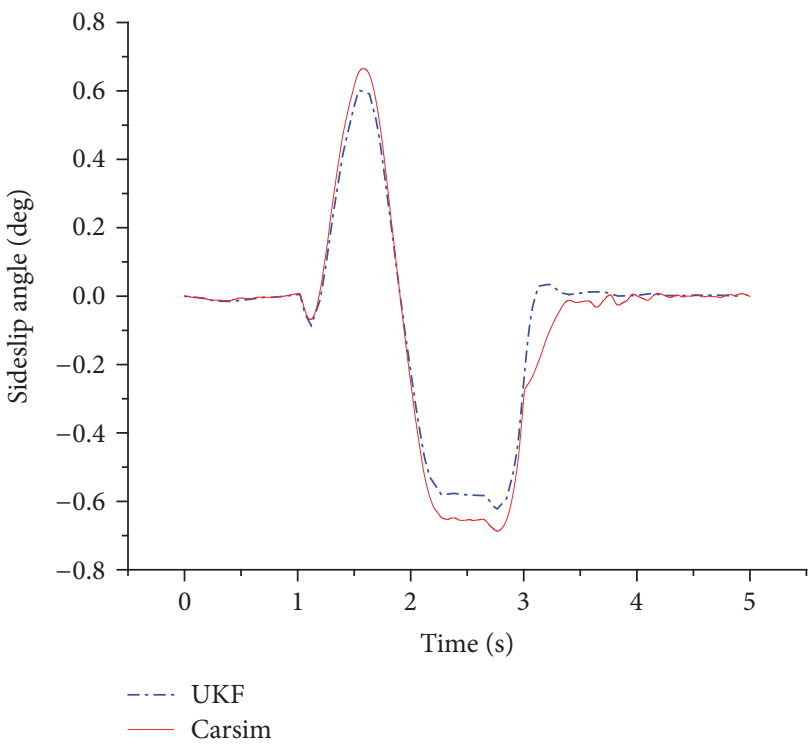

FIGURE 10: Estimation results comparison of sideslip angle.

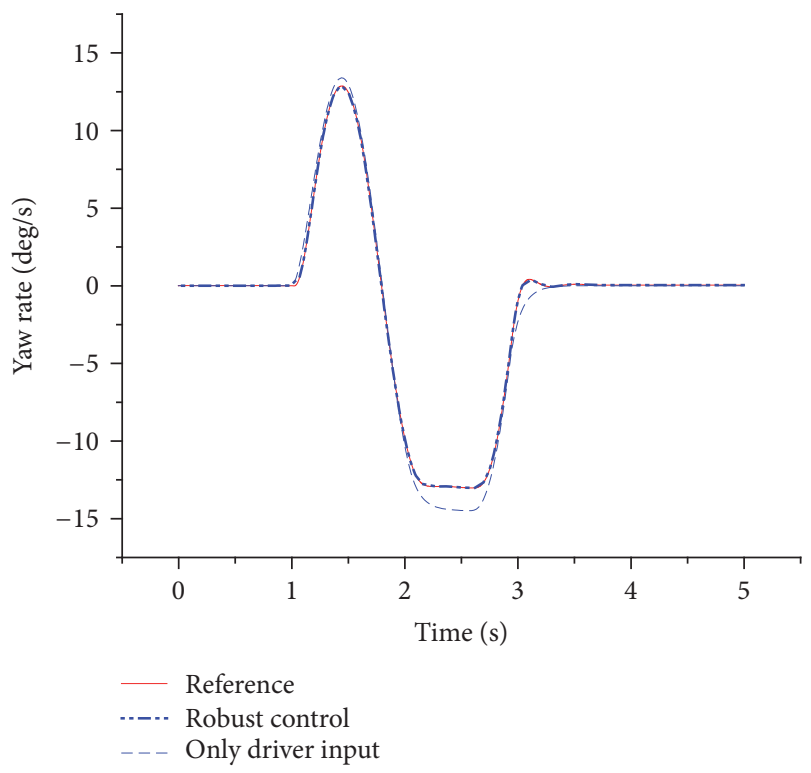

FIGURE 11: Estimation results comparison of yaw rate.

can constrain the sideslip angle effectively. This means that the robust controller could improve the vehicle stability especially when vehicle is in high speed or sharp curve turning condition.

Figure 13 shows the lateral acceleration of vehicle mass center comparison. It is well known that large lateral acceleration brings more load transfer and could lead to inadequate using of the tire forces. With the proposed control algorithm, the lateral acceleration is smaller compared with no control. So the proposed controller has potentials in the improvement of vehicle stability.

The proposed systems help improve stability and response at high speed and make the vehicle have a tendency to understeer. An understeering vehicle is self-stabilizing through its 


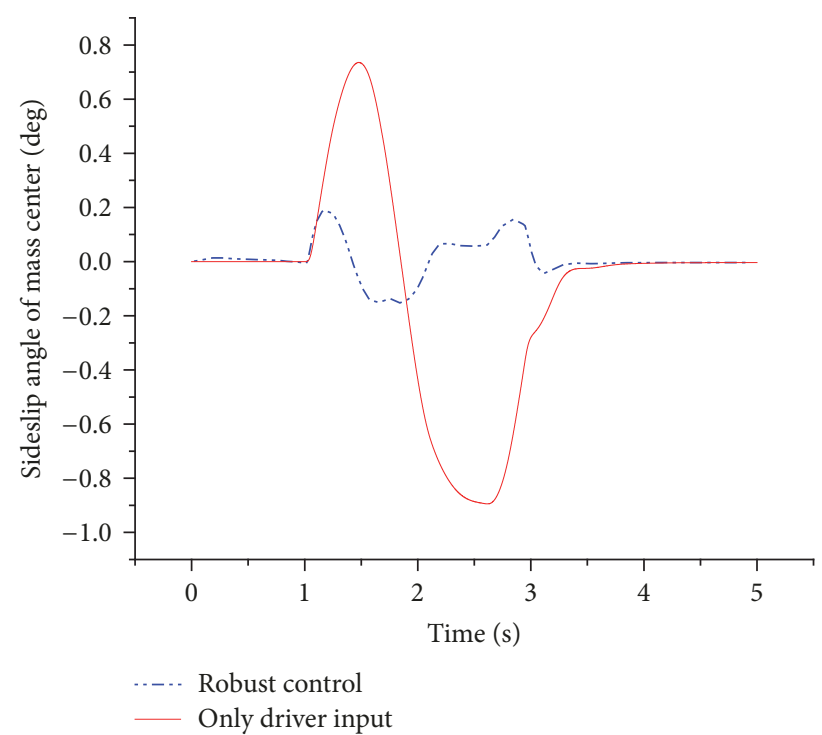

FIGURE 12: Sideslip angle in mass center comparison.

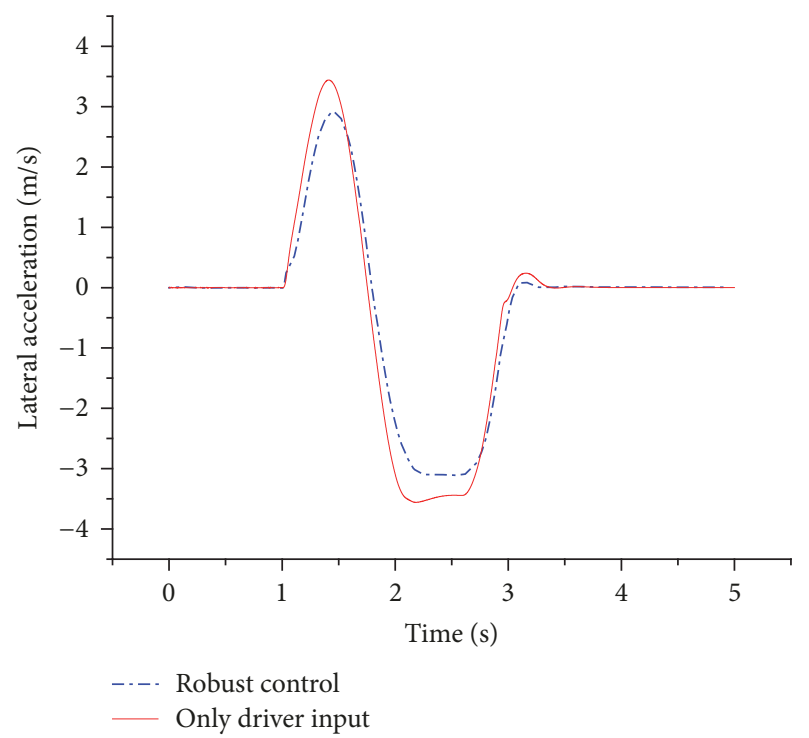

FIGURE 13: Lateral acceleration in mass center comparison.

tendency to return to straight ahead motion after any external disturbances. Figures 14 and 15 plotted the front wheel active steering angle and the total added yaw moment from the controller.

Figure 16 presents tire force allocation results from the total added yaw moment. It can be found that, combined with the active front steering and motor in-wheel actuators, the vehicle stability is improved significantly. And the controller integrates the longitudinal and lateral dynamic to make good using of tire forces so as to avoid the saturation of tire forces.

\section{Conclusion and Future Works}

This paper proposed the $H_{\infty}$ robust control strategy for motor in-wheel four wheels driving electric vehicles. The

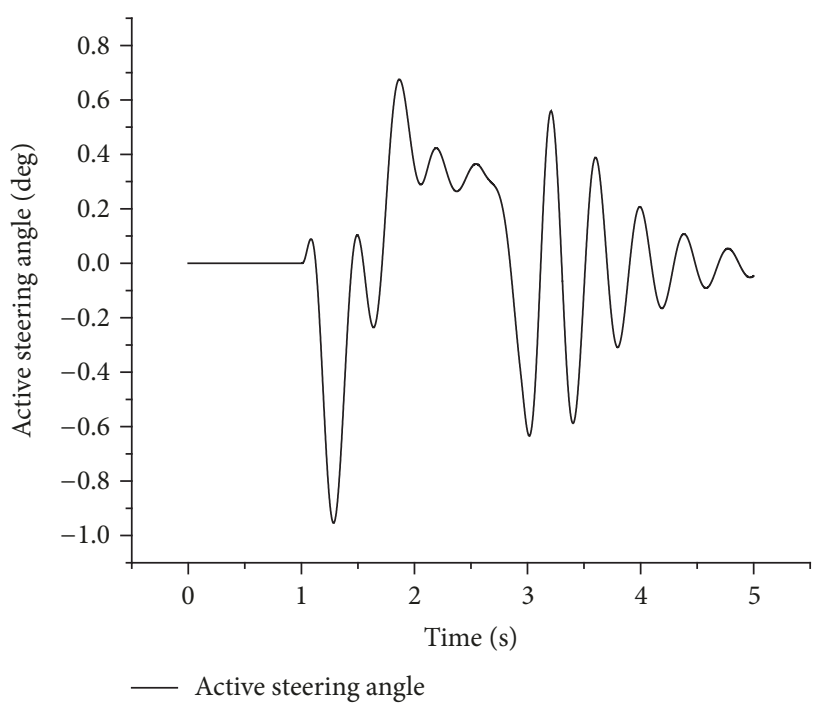

FIGURE 14: Active front steering correction angle.

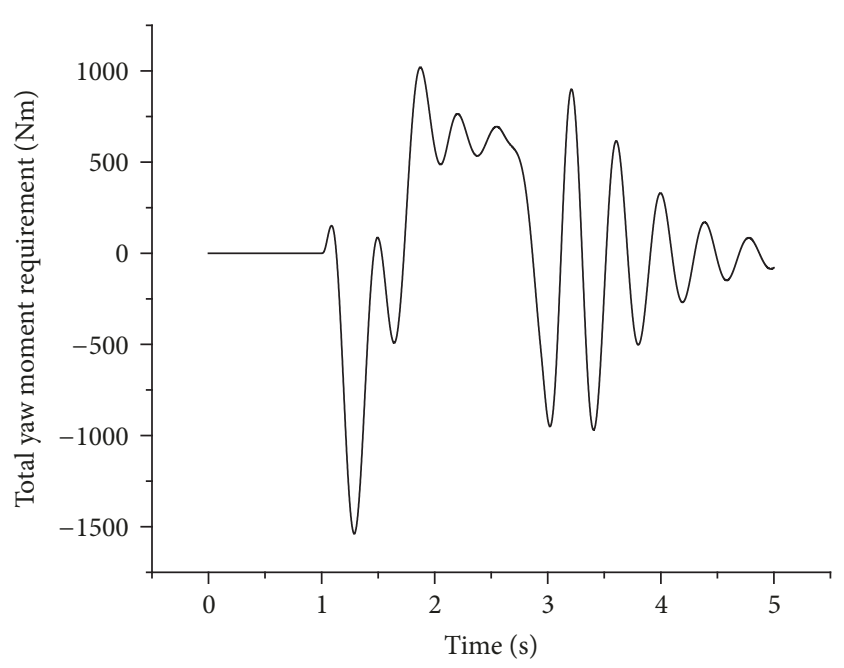

FIgURE 15: The total yaw moment requirement from controller.

proposed control strategy integrates the longitudinal and lateral dynamic control through the active front steering and yaw moment control systems considering the saturation characteristics of tire forces. An upper level control system is designed by using $H_{\infty}$ robust control strategy combined with the online sideslip angle observer to specify a desired yaw moment and correction front steering angle to work on the EVs. The four-wheel torque is obtained optimally by the wheel torque distribution control algorithm. According to the simulation results, it can be asserted that the robust control algorithm could improve vehicle maneuverability and high speed stability. This is very important for vehicle safety. The future works will be focusing on how to distribute the torque smoothly among the axles according to different road friction coefficients or driving situations. 


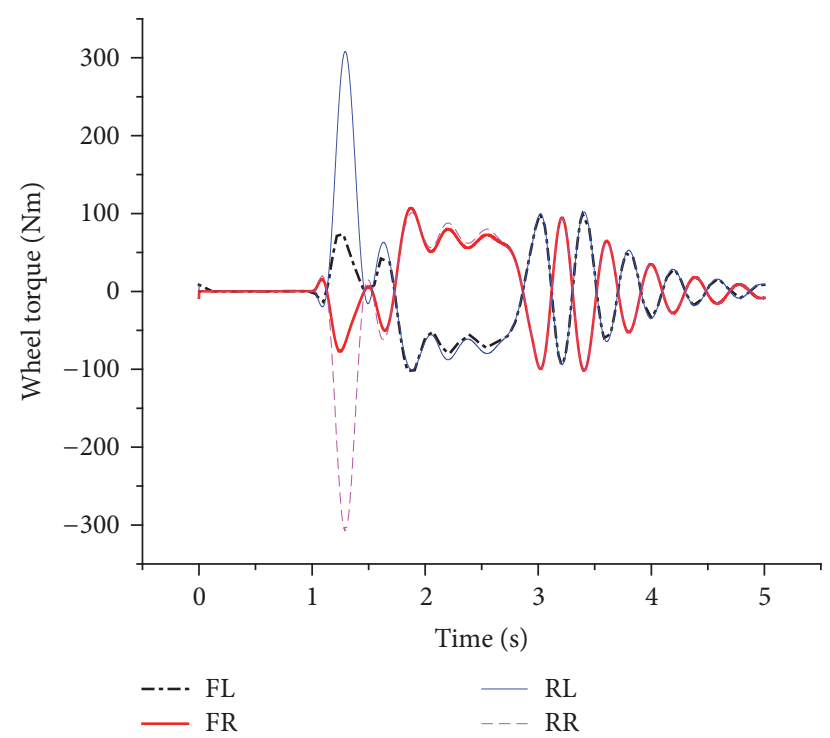

FIGURE 16: Four-wheel torque control input for in-wheel motors.

\section{Nomenclature}

$\begin{array}{ll}\beta: & \text { Sideslip angle } \\ r: & \text { Yaw rate } \\ \delta_{\text {sw }}: & \text { Steering wheel angle from driver } \\ \delta_{c}: & \text { Wheel steering angle from active steering } \\ & \text { controller } \\ u, m: & \text { Vehicle velocity/mass } \\ F_{y f}, F_{y r}: & \text { Lateral force of front/rear tires } \\ a_{y}: & \text { Lateral acceleration of vehicle mass center } \\ I_{z}: & \text { Yaw moment of inertia } \\ a, b: & \text { Distance from the vehicle mass center of } \\ & \text { gravity to front/rear axle } \\ B_{f}, B_{r}: & \text { Track width of front/rear axle } \\ k_{f}, k_{r}: & \text { Cornering stiffness at front/rear tire } \\ T_{i j}: & \text { Motor torque on the wheel } \\ T_{n}: & \text { Close loop transfer function } \\ L_{n}: & \text { Open loop transfer function. }\end{array}$

\section{Conflicts of Interest}

The authors declare that there are no conflicts of interest regarding the publication of this paper.

\section{Acknowledgments}

This work is financially supported by the Fundamental Research Funds for the Central Universities (no. 2015ZCQGX-01) and China Postdoctoral Science Foundation (2016M600048).

\section{References}

[1] E. Esmailzadeh, A. Goodarzi, and G. R. Vossoughi, "Optimal yaw moment control law for improved vehicle handling," Mechatronics, vol. 13, no. 7, pp. 659-675, 2003.
[2] C. Gu, J. Zhu, and X. Chen, "A Novel E-DVA Module Synthesis Featuring of Synergy between Driving and Vibration Attenuation," Shock and Vibration, vol. 2016, Article ID 8464317, 2016.

[3] B. Li, A. Goodarzi, A. Khajepour, S.-K. Chen, and B. Litkouhi, "An optimal torque distribution control strategy for four-independent wheel drive electric vehicles," Vehicle System Dynamics, vol. 53, no. 8, pp. 1172-1189, 2015.

[4] D. Tan, Q. Wang, and Y. Wu, "Modal Analysis of In-Wheel Motor-Driven Electric Vehicle Based on Bond Graph Theory," Shock and Vibration, vol. 2017, Article ID 6459154, 2017.

[5] W. Christian, D. Odenthal, and M. Sailer, "Control system for a motor vehicle having an electronic control unit by which the drive torque of a drive unit can be variably distributed, as required, on at least two axles," vol. 9, pp. 186-988, Patent, USA, 2015.

[6] T. Ringler, J. Steiner, R. Belschner, and B. Hedenetz, "Increasing System Safety for By-Wire Applications in Vehicles by Using a Time Triggered Architecture," in Computer Safety, Reliability and Security, vol. 1516 of Lecture Notes in Computer Science, pp. 243-253, Springer Berlin Heidelberg, Berlin, Heidelberg, 1998.

[7] M. Suzuki, K. Okada, K. Sakai, and Y. Makino, "Development of an In-Wheel Motor Axle Unit," Tech. Rep. 75, 2007.

[8] M. Kamachi et al., "Improvement of Vehicle Dynamic Performance by Means of In-Wheel Electric Motors, Mitsubishi Motors Technical Review," Tech. Rep. 18, 2006.

[9] R. S. Zhou and F. Hashimoto, "Highly compact electric drive for automotive applications," SAE Technical Papers, 2004.

[10] C. Lin and Z. Xu, "Wheel torque distribution of four-wheeldrive electric vehicles based on multi-objective optimization," Energies, vol. 8, no. 5, pp. 3815-3831, 2015.

[11] Y. Yang, K. A. Ali, J. Roeleveld, and A. Emadi, "State-of-the-art electrified powertrains - hybrid, plug-in, and electric vehicles," International Journal of Powertrains, vol. 5, no. 1, p. 1, 2016.

[12] A. Khaligh and Z. Li, "Battery, ultracapacitor, fuel cell, and hybrid energy storage systems for electric, hybrid electric, fuel cell, and plug-in hybrid electric vehicles: state of the art," IEEE Transactions on Vehicular Technology, vol. 59, no. 6, pp. 28062814, 2010.

[13] J. Y. Yong, V. K. Ramachandaramurthy, K. M. Tan, and N. Mithulananthan, "A review on the state-of-the-art technologies of electric vehicle, its impacts and prospects," Renewable \& Sustainable Energy Reviews, vol. 49, pp. 365-385, 2015.

[14] B. Wang, X. Huang, J. Wang, X. Guo, and X. Zhu, "A robust wheel slip ratio control design combining hydraulic and regenerative braking systems for in-wheel-motors-driven electric Vehicles," Journal of The Franklin Institute, vol. 352, no. 2, pp. 577-602, 2015.

[15] K. Nam, H. Fujimoto, and Y. Hori, "Lateral stability control of in-wheel-motor-driven electric vehicles based on sideslip angle estimation using lateral tire force sensors," IEEE Transactions on Vehicular Technology, vol. 61, no. 5, pp. 1972-1985, 2012.

[16] Z. Shuai, H. Zhang, J. Wang, J. Li, and M. Ouyang, "Combined AFS and DYC control of four-wheel-independent-drive electric vehicles over CAN Network with time-varying delays," IEEE Transactions on Vehicular Technology, vol. 63, no. 2, pp. 591-602, 2014.

[17] H. Zhao, B. Gao, B. Ren, H. Chen, and W. Deng, "Model predictive control allocation for stability improvement of fourwheel drive electric vehicles in critical driving condition," IET Control Theory \& Applications, vol. 9, no. 18, pp. 2688-2696, 2015. 
[18] H. Her, E. Joa, K. Yi, and K. Kim, "Integrated chassis control for optimized tyre force coordination to enhance the limit handling performance," Proceedings of the Institution of Mechanical Engineers, Part D: Journal of Automobile Engineering, vol. 230, no. 8, pp. 1011-1026, 2016.

[19] H. Zhang and J. Wang, "Vehicle lateral dynamics control through AFS/DYC and robust gain-scheduling approach," IEEE Transactions on Vehicular Technology, 2015.

[20] T. van der Sande, P. Zegelaar, I. Besselink, and H. Nijmeijer, "A robust control analysis for a steer-by-wire vehicle with uncertainty on the tyre forces," Vehicle System Dynamics, vol. 54, no. 9, pp. 1247-1268, 2016.

[21] J. Wu, Y. Liu, F. Wang, C. Bao, Q. Sun, and Y. Zhao, "Vehicle active steering control research based on two-dof robust internal model control," Chinese Journal of Mechanical Engineering, vol. 29, no. 4, pp. 739-746, 2016.

[22] H. Ren, T. Shim, J. Ryu, and S. Chen, "Development of effective bicycle model for wide ranges of vehicle operations," $S A E$ Technical Papers, vol. 1, 2014.

[23] S. Skogestad and I. Postlethwaite, Multivariable feedback control: analysis and design, JohnWiley Sons, Chichester, 2005.

[24] E. Ono, S. Hosoe, H. D. Tuan, and S. Doi, "Bifurcation in vehicle dynamics and robust front wheel steering control," IEEE Transactions on Control Systems Technology, vol. 6, no. 3, pp. 412-420, 1998.

[25] C. Yang, X. Jiao, L. Li, Y. Zhang, L. Zhang, and J. Song, "Robust coordinated control for hybrid electric bus with single-shaft parallel hybrid powertrain," IET Control Theory \& Applications, vol. 9, no. 2, pp. 270-282, 2015.

[26] S. J. Julier and J. K. Uhlmann, "A new extension of the Kalman filter to nonlinear systems," in Proceedings of the Signal Processing, Sensor Fusion, and Target Recognition VI, vol. 3068 of Proceedings of SPIE, pp. 182-193, Orlando, Fla, USA, 1997.

[27] W. Eric and R. van der Merwe, Kalman Filtering and Neural Networks. Chapter 7: The Unscented Kalman Filter, Chapter 7, Wiley Publishin, New Jersey, USA, 2001.

[28] S. Sigurd and I. Postlethwaite, Multivariable feedback control: analysis and design. Vol. 2. New York: Wiley, 2007.

[29] R. Wang, C. Hu, Z. Wang, F. Yan, and N. Chen, "Integrated optimal dynamics control of 4WD4WS electric ground vehicle with tire-road frictional coefficient estimation," Mechanical Systems and Signal Processing, vol. 60, pp. 727-741, 2015.

[30] J. Yoon, W. Cho, B. Koo, and K. Yi, "Unified chassis control for rollover prevention and lateral stability," IEEE Transactions on Vehicular Technology, vol. 58, no. 2, pp. 596-609, 2009.

[31] L. haoqi, Research on Hierarchical Chassis Integrated Control Based on Model Predictive Control Method. Master thesis [Master, thesis], Jilin University, 2011. 


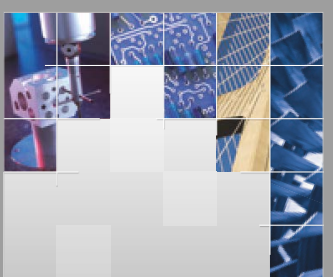

\section{Enfincering}
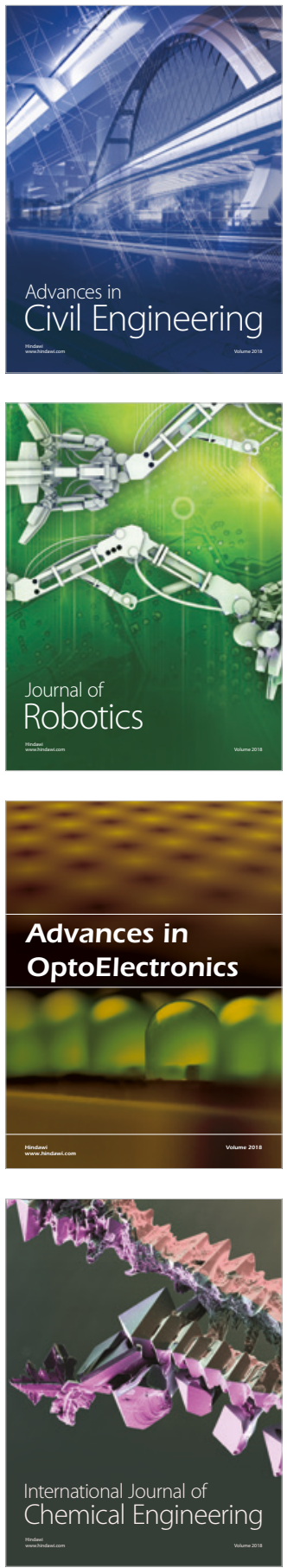

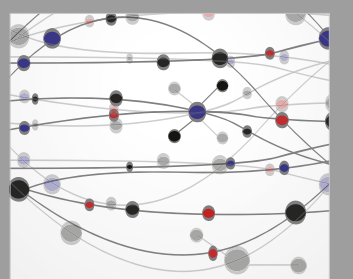

\section{Rotating \\ Machinery}

The Scientific World Journal

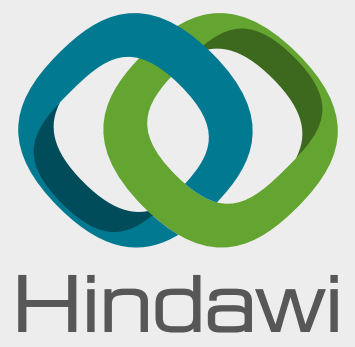

Submit your manuscripts at

www.hindawi.com
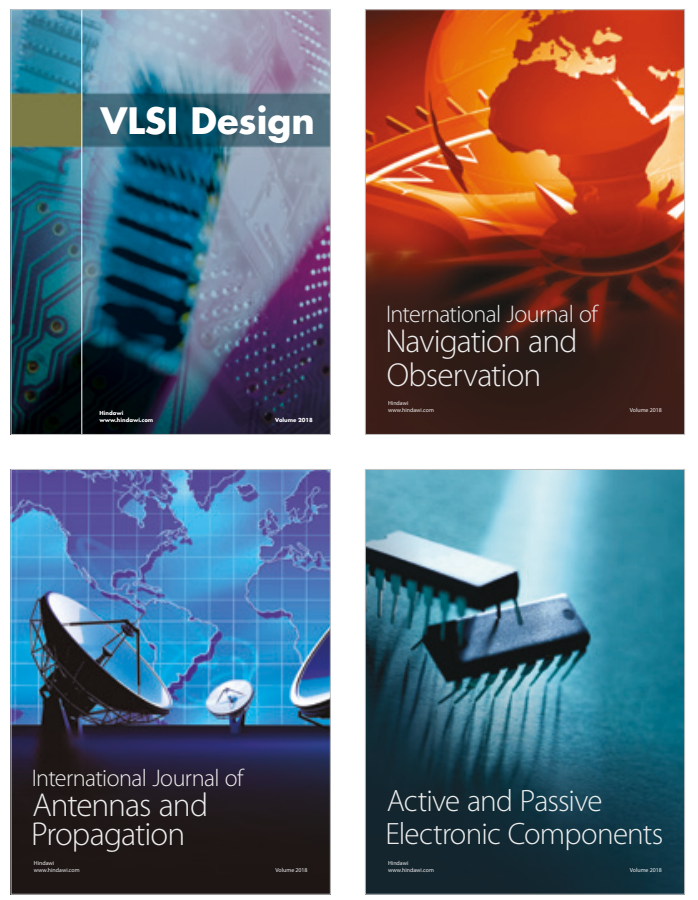
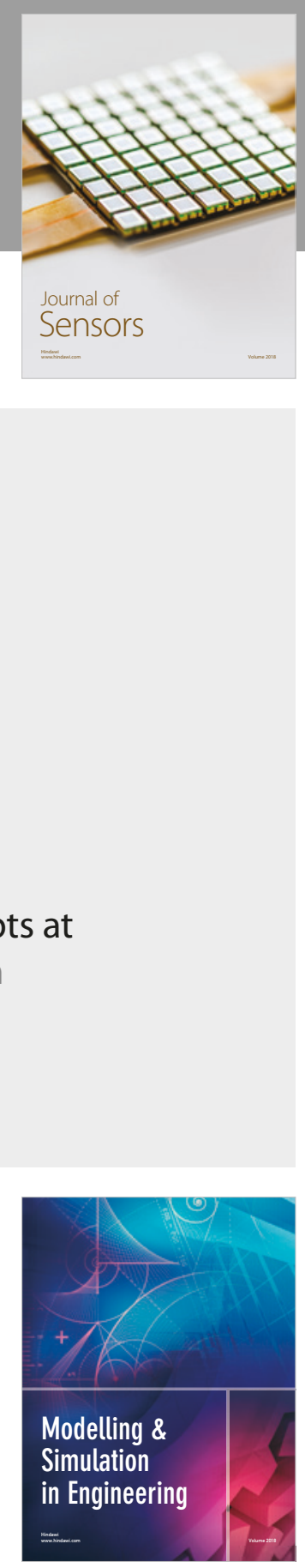

\section{Advances \\ Multimedia}
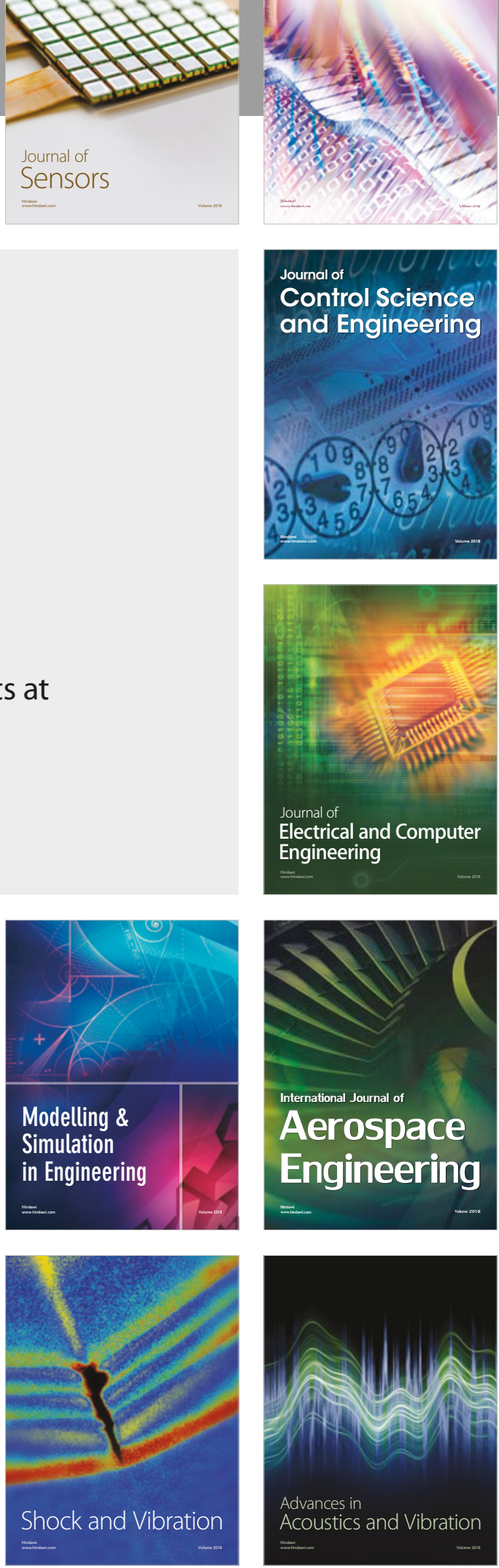